\title{
Vitamin D receptor polymorphisms or serum levels as key drivers of breast cancer development? The question of the vitamin D pathway
}

\author{
Dino Amadori' ${ }^{1}$, Patrizia Serra², Nestory Masalu ${ }^{3}$, Akwilina Pangan $^{3}$, Emanuela $^{2}$ \\ Scarpi $^{2}$, Aloyce Maria Bugingo ${ }^{3}$, Deogratias Katabalo ${ }^{3}$, Toni Ibrahim ${ }^{4}$, Alberto \\ Bongiovanni ${ }^{4}$, Giacomo Miserocchi ${ }^{4}$, Chiara Spadazzi ${ }^{4}$, Chiara Liverani ${ }^{4}$, Valentina \\ Turri $^{5}$, Rosanna Tedaldi ${ }^{1}$, Laura Mercatali ${ }^{4}$ \\ ${ }^{1}$ Department of Medical Oncology, Istituto Scientifico Romagnolo per lo Studio e la Cura dei Tumori (IRST) IRCCS, Meldola, \\ Italy \\ ${ }^{2}$ Unit of Biostatistics and Clinical Trials, Istituto Scientifico Romagnolo per lo Studio e la Cura dei Tumori (IRST) IRCCS, \\ Meldola, Italy \\ ${ }^{3}$ Department of Oncology, Bugando Medical Center, Mwanza, Tanzania, Africa \\ ${ }^{4}$ Osteoncology and Rare Tumors Center, Istituto Scientifico Romagnolo per lo Studio e la Cura dei Tumori (IRST) IRCCS, \\ Meldola, Italy \\ ${ }^{5}$ Healthcare Administration, Istituto Scientifico Romagnolo per lo Studio e la Cura dei Tumori (IRST) IRCCS, Meldola, Italy \\ Correspondence to: Dino Amadori, email: dino.amadori@irst.emr.it \\ Keywords: Africans, caucasians, 25(OH)D, vitamin D pathway, vitamin D binding protein \\ Received: September 27, $2016 \quad$ Accepted: December 15, $2016 \quad$ Published: January 04, 2017
}

\section{ABSTRACT}

As total vitamin D levels are often lower in black than in white Americans, the former are frequently classified as vitamin D-deficient. To fully understand African vitamin D (25(OH)D) status, other factors should be considered, e.g. vitamin D blood carrier, vitamin D-binding protein (DBP), vitamin D receptor (VDR) and DBP polymorphisms. A prospective study on an indigenous black Tanzanian and a Caucasian Italian population was performed on $\mathbf{5 0}$ healthy donors from both populations and 35 Caucasian and 18 African breast cancer patients. 25(OH)D and DBP serum levels were analyzed by ELISA. A1012G, Cdx2 and Fok1 VDR polymorphisms and DBP polymorphisms rs4588 and rs7041 were genotyped by real-time PCR. Vitamin D and DBP levels were lower in healthy African donors than in Caucasians. Africans had a significantly higher frequency of $\mathrm{AA}$ and $\mathrm{CC}$ for $\mathrm{Cdx} 2$ and Fok1 polymorphisms, respectively. These allelic variants were related to a higher transcription of VDR gene and a higher activity of VDR receptor. With regard to polymorphism distribution, Africans showed innate higher levels and activity of VDR. We conclude that a strengthening of the vitamin $D$ pathway could have a protective role against the development of breast cancer in the African population.

\section{INTRODUCTION}

Ethnic differences play a role in circulating levels of serum vitamin D: black Americans are more likely to have lower levels of circulating 25(OH)D than white Americans, as darker skin pigmentation is associated with a slighter increase in serum concentration after a given amount of UVB exposure [1-5]. Vitamin D derived from diet and sunlight-driven synthesis in the skin is converted by the liver into its circulating form 25 -hydroxyvitamin
D $(25(\mathrm{OH}) \mathrm{D}) .25(\mathrm{OH}) \mathrm{D}$ is thus transformed into the active form 1,25-dihydroxyvitamin D3 (calcitriol) in the kidney and in target tissues, including breast cancer (BC) cells [6]. Its classical physiological function is to regulate bone development and extracellular calcium concentration in the organism. The nonclassic functions of vitamin D range from hypertension and disturbed muscle function to susceptibility to infections autoimmune diseases and cancer. At a cellular level vitamin D cancer-related activity includes induction to 
cell differentiation, inhibition of proliferation by cellcycle blockage or interference with signaling by growth factors, inducing apoptosis, stimulation of DNA damage repair, prevention of tumor angiogenesis, and inhibition of metastasis [7-9]. The observations of this spread of functions indicated a inhibitory role of vitamin D in cancer development.

In accordance with these data, vitamin $\mathrm{D}$ deficiency is common in patients diagnosed with $\mathrm{BC}$, associating with worse prognosis and outcome [10]. In particular epidemiological data reported an inverse correlation between the risk of developing BC and serum 25(OH)D levels [11-13].

For these reasons the role of vitamin D supplementation in cancer prevention or in early-stage cancer patients has been investigated. Although most clinical data derive from studies showing a potential benefit of vitamin D supplementation in colon and breast cancer patients (BCPs), definitive randomized clinical trials of adequate size and duration with sufficient doses of vitamin D are still lacking [14-16].

Information on the vitamin $\mathrm{D}$ pathway activity is obtained not only through the observation of serum $25(\mathrm{OH}) \mathrm{D}$ levels. Vitamin D-binding protein $(\mathrm{DBP})$ is the main blood carrier of vitamin D, binding around $85-90 \%$ of total circulating vitamin $\mathrm{D}$. The vitamin-D fraction unbound to DBP is bound to albumin, with $<1 \%$ of total free vitamin D in blood [17].

According to the theory of the free hormones, vitamin D may enter target cells and work only when it is unbound to protein carriers [18-19]. DBP may thus be considered an important regulator of vitamin D activity. Inside the cell, most of the known effects of vitamin D are mediated via binding of the active forms of vitamin $\mathrm{D}$ to the vitamin $\mathrm{D}$ receptor (VDR), that is a regulator of the transcription expressed in almost all tissues, including normal breast and most BCs [20-22]. Common genetic polymorphisms in $V D R$ and in $D B P$ produce variant proteins which generate a different activity of the vitamin D pathway, especially in their affinity to vitamin D [23-24]. The prevalence of these polymorphisms differs among racial groups [8, 25-26]. Only Serum 25(OH)D levels have been evaluated in clinical practice $[20,25$, 26], with a lack of information on the activation status of vitamin D.

Besides having lower levels of vitamin D, African Americans have a lower incidence of BC than Caucasians. The same trend has been observed in Native Africans. At the same time, as reported in our previous paper, [27] Tanzanian and Italian BCs present different histopathological and biomolecular characteristics: African patients have a higher frequency of negative prognostic markers, such as poor differentiation grade, ER-negative status, high proliferation index or advanced stage at diagnosis [28].
A comprehensive study on vitamin D pathway status and differences in African and Caucasian populations and their implication on BC is still missing. Moreover, most of the information available for Africans is gathered from studies on black Americans and only a few on native Africans [29-34].

We thus conducted a study in collaboration with the Bugando Medical Centre of Mwanza (Tanzania, Africa), to assess the levels of vitamin D and DBP and their relation to VDR and DBP protein genotypes between a native African and a Caucasian population, with the aim to study the differences in the vitamin D pathway between the two populations and their implications in cancer.

\section{RESULTS}

\section{5(OH)D and DBP circulating levels}

\section{Africans vs. Caucasians}

Levels of 25(OH)D and DBP were significantly lower $(P=0.003$ and $P<0.0001$, respectively $)$ in African than in Caucasian HDs, when considered as continuous variables (Table 1). In particular DBP was about threefold lower in African HDs (Table 1). DBP median levels were about threefold lower $(P<0.0001)$ also in African BCPs, with no change in $25(\mathrm{OH}) \mathrm{D}$ median levels.

A different distribution of vitamin $\mathrm{D}$ and DBP ( $P=0.0073$ and $<0.0001$, respectively) was observed between African and Caucasian HDs, when considered as discrete variables with respect to the classes of vitamin $\mathrm{D}$ and DBP levels reported in the materials and methods section. In particular, a higher percentage of African HDs showed vitamin D levels $<20 \mathrm{ng} / \mathrm{ml}$ (38\% vs. 8\%, respectively). Similarly, most African HDs (64\%) had low levels of DBP, compared to Caucasian HDs $(0 \%, P$ $<0.001)$. These differences were lost when comparing BCPs (Table 2).

The Spearman's analyses revealed a direct correlation between 25(OH)D and DBP levels when considered as continuous variables. The increase in $25(\mathrm{OH}) \mathrm{D}$ was associated with an increase in DBP levels in the overall case series $\left(\mathrm{r}_{\mathrm{s}}=0.24 ; P=0.003\right)$, in the Caucasian case series $\left(\mathrm{r}_{\mathrm{s}}=0.24 ; P=0.018\right)$ and in the entire healthy subgroup $\left(\mathrm{r}_{\mathrm{s}}=0.31 ; P=0.002\right)$.

\section{HDs vs BCP within each population}

Unlike DBP, vitamin D median levels were significantly higher in African BCPs than in HDs (Table 1) $(P=0.013)$. Level distribution of circulating markers was not significantly different between cases and controls, when considered as discrete variables (Table 2). Both markers showed no statistical difference in the Caucasian population. 
Table 1: 25(OH)D and DBP in BCPs and HDs

Median values (range) of 25(OH)D and DBP in BCPs and HDs

\begin{tabular}{|c|c|c|c|c|c|c|}
\hline & \multicolumn{3}{|c|}{ Healthy } & \multicolumn{2}{|c|}{ Patients } & \multirow[b]{2}{*}{$P^{a}$} \\
\hline & Africans $(n=50)$ & Caucasians $(n=50)$ & $P^{a}$ & Africans $(n=18)$ & Caucasians $(n=35)$ & \\
\hline $\begin{array}{l}\text { 25(OH)D } \\
(\mathrm{ng} / \mathrm{ml})\end{array}$ & $22.50(5-94)$ & $28.32(11.26-60.70)$ & 0.0030 & $33.50(14-65)$ & $27.01(11.00-53.22)$ & 0.1450 \\
\hline $\begin{array}{l}\mathrm{DBP}(\mu \mathrm{g} / \\
\mathrm{ml})\end{array}$ & $75.13(23.34-194.75)$ & $219.62(96.32-738.06)$ & $<0.0001$ & $\begin{array}{c}87.89(13.69- \\
197.44)\end{array}$ & $\begin{array}{c}227.98(79.41- \\
395.71)\end{array}$ & $<0.0001$ \\
\hline
\end{tabular}

Africans: BCPs vs. HDs: 25(OH)D: $P^{\mathrm{a}}=0.013$ DBP: $P^{\mathrm{a}}=0.5150$.

Caucasians: BCPs vs. HDs: 25(OH)D: $P^{\mathrm{a}}=0.399$ DBP: $P^{\mathrm{a}}=0.5490$.

${ }^{\mathrm{a}}$ median test.

Table 2: 25(OH)D and DBP considered as discrete variables in BCPs and HDs

\begin{tabular}{|c|c|c|c|c|}
\hline \multirow{2}{*}{ Marker } & \multicolumn{2}{|c|}{ Africans } & \multicolumn{2}{|c|}{ Caucasians } \\
\hline & Healthy No. (\%) & Patients No. (\%) & Healthy No. (\%) & Patients No. (\%) \\
\hline \multicolumn{5}{|c|}{ 25(OH)D (ng/ml) } \\
\hline$\leq \mathbf{2 0}$ & $19(38.0)$ & $4(22.2)$ & $4(8.0)$ & $6(17.1)$ \\
\hline 20.1-30 & $19(38.0)$ & $4(22.2)$ & $27(54.0)$ & $16(45.7)$ \\
\hline $30.1-50$ & $9(18.0)$ & $9(50.0)$ & $15(30.0)$ & $11(31.1)$ \\
\hline$>\mathbf{5 0}$ & $3(6.0)$ & $1(5.6)$ & $4(8.0)$ & $2(5.7)$ \\
\hline \multicolumn{5}{|c|}{$\mathrm{DBP}(\mu \mathrm{g} / \mathrm{ml})$} \\
\hline$\leq 98$ & $32(64.0)$ & $9(56.2)$ & 0 & $2(5.9)$ \\
\hline 98-198 & $18(36.0)$ & $7(43.8)$ & $22(47.8)$ & $11(32.3)$ \\
\hline$>198$ & 0 & 0 & $24(52.2)$ & $21(61.8)$ \\
\hline
\end{tabular}

\section{SNP distribution}

The genotype distributions of all selected polymorphisms from HDs and BCPs in both populations were analyzed by ethnicity for the Hardy-Weinberg Equilibrium. A1012G and Cdx2 polymorphisms were in linkage disequilibrium in both populations and therefore analyzed as haplotypes (three variants: Haplo1: CG, Haplo2: TG, and Haplo 3 TA) (Table 3). DBP haplotype analyses were performed only for Caucasian subjects, as the Africans reported no DBP polymorphisms in linkage disequilibrium (Haplo1: CG, Haplo2: CT, Haplo 3 AT and Haplo 4, others) (Table 3). We compared the allelic distributions of polymorphisms in our case series with those reported in European Caucasian and African populations extrapolated by the 1000 Genomes database (Table 4) [35]. We compared our Caucasian case series with an Italian Tuscany population, and our Tanzanian population with an American ancestry population (ASW) and an African population (YRI). The allelic frequency of all analyzed polymorphisms were homogeneous with the 1000 Genomes dataset for the Caucasian population. We observed a statistically significant difference in the African population for $\mathrm{Cdx} 2$ and $\mathrm{rs} 4588$, although our data showed a percentage trend similar to that reported by the 1000 Genome dataset (Table 4).

\section{VDR SNPs}

\section{Africans vs Caucasians}

African HDs showed a higher frequency of TT and AA homozygosity in A1012G and Cdx2 than Caucasian HDs $(P<0.0001$ for both SNPs). Moreover, Africans had a higher $\mathrm{CC}$ frequency than Caucasians for Fok1 polymorphism ( $70 \%$ vs. $46 \%$, respectively; $P=0.00253$ ) (Table 5). We observed the same significant trends for A1012G and Cdx2 polymorphisms in BCPs: Africans showed $100 \%$ homozygosity for both SNPs (Table 5) compared to $34.3 \%$ and $11.4 \%$ observed in Caucasians. Fok1, instead, was similarly distributed in BCPs. The A1012G/Cdx2 haplotype was differently distributed among African and Caucasian HDs and BCPs $(P<0.0001$ for both subgroups); In particular, 78\% of African HDs and $100 \%$ of BCPs had haplotype TA/TA, compared to $8 \%$ and $14 \%$ of Caucasian HDs and BCPs, respectively (Table 3). 
Table 3: Distribution of haplotypes in both populations

\begin{tabular}{|c|c|c|c|c|}
\hline \multirow[b]{2}{*}{ Haplotype } & \multicolumn{2}{|c|}{ Africans } & \multicolumn{2}{|c|}{ Caucasians } \\
\hline & $\begin{array}{l}\text { Healthy } \\
\text { No. (\%) }\end{array}$ & $\begin{array}{l}\text { Patients } \\
\text { No. }(\%)\end{array}$ & $\begin{array}{l}\text { Healthy } \\
\text { No. (\%) }\end{array}$ & $\begin{array}{l}\text { Patients } \\
\text { No (\%) }\end{array}$ \\
\hline \multicolumn{5}{|c|}{$\mathrm{A} 1012 \mathrm{~A} / \mathrm{Cdx} 2$} \\
\hline CG/CG & $1(2)$ & $0(0)$ & $5(10)$ & $4(11.4)$ \\
\hline CG/TG & $1(2)$ & $0(0)$ & $15(30)$ & $13(37.1)$ \\
\hline CG/TA & $3(6)$ & $0(0)$ & $10(20)$ & $6(17.1)$ \\
\hline TG/TG & $0(0)$ & $0(0)$ & $6(12)$ & $3(8.6)$ \\
\hline TG/TA & $6(12)$ & $0(0)$ & $10(20)$ & $5(14.3)$ \\
\hline TA/TA & $39(78)$ & $18(100)$ & $4(8)$ & $4(11.4)$ \\
\hline \multicolumn{5}{|c|}{ rs7041/rs4588 } \\
\hline CG/CG & - & - & $16(32)$ & $12(34.3)$ \\
\hline $\mathrm{CT} / \mathrm{CT}$ & - & - & $1(2)$ & $1(2.9)$ \\
\hline CT/other & - & - & $0(0)$ & $1(2.9)$ \\
\hline AT/CG & - & - & $12(24)$ & $5(14.3)$ \\
\hline $\mathbf{A T} / \mathbf{C T}$ & - & - & $7(14)$ & $5(14.3)$ \\
\hline AT/AT & - & - & $2(4)$ & $1(2.86)$ \\
\hline AT/other & - & - & $12(24)$ & $10(41.1)$ \\
\hline
\end{tabular}

Table 4: Comparison of allelic frequencies (\%) of SNPs with those reported in the 1000 Genome Dataset

\begin{tabular}{|c|c|c|c|c|c|c|c|c|c|c|c|c|c|c|c|c|c|c|c|c|}
\hline & & \multicolumn{3}{|c|}{ A1012G } & \multicolumn{4}{|c|}{ CDX2 } & \multicolumn{4}{|c|}{ FOK1 } & \multicolumn{4}{|c|}{ rs4588 } & \multicolumn{3}{|c|}{ rs7041 } & \multirow[b]{2}{*}{$\mathrm{p}$} \\
\hline & & TSI* & C. S. & $\mathrm{p}$ & & TSI & C. S. & $\mathrm{p}$ & & TSI & C. S. & $\mathrm{p}$ & & TSI & C. S. & $\mathrm{p}$ & & TSI & C. S. & \\
\hline \multirow[t]{3}{*}{ Caucasians } & $\mathrm{C}$ & 42 & 35 & & $\mathrm{C}$ & 75 & 72 & & G & 61 & 65 & & $\mathrm{G}$ & 79 & 75 & & $\mathrm{C}$ & 55 & 57 & \\
\hline & $\mathrm{T}$ & 58 & 65 & 0.31 & $\mathrm{~T}$ & 25 & 28 & 0.632 & A & 38 & 35 & 0.621 & $\mathrm{~T}$ & 21 & 25 & 0.503 & A & 45 & 43 & 0.776 \\
\hline & \multicolumn{3}{|c|}{ AWS* } & \multicolumn{4}{|c|}{ AWS } & \multicolumn{5}{|c|}{ AWS } & \multicolumn{3}{|c|}{ AWS } & \multicolumn{5}{|c|}{ AWS } \\
\hline \multirow[t]{5}{*}{ Africans } & $\mathrm{C}$ & 12 & 6 & & $\mathrm{C}$ & 28 & 13 & & G & 80 & 84 & & G & 78 & 91 & & $\mathrm{C}$ & 16 & 8 & \\
\hline & $\mathrm{T}$ & 88 & 94 & 0.139 & $\mathrm{~T}$ & 72 & 87 & 0.009 & A & 20 & 16 & 0.463 & $\mathrm{~T}$ & 22 & 8 & 0.006 & A & 84 & 92 & 0.082 \\
\hline & & YRI* & & & & YRI & & & & YRI & & & & YRI & & & & YRI & & \\
\hline & $\mathrm{C}$ & 1 & & & $\mathrm{C}$ & 2 & & & G & 81 & & & G & 96 & & & $\mathrm{C}$ & 3 & & \\
\hline & $\mathrm{T}$ & 99 & & 0.118 & $\mathrm{~T}$ & 98 & & 0.005 & A & 19 & & 0.578 & $\mathrm{~T}$ & 3 & & 0.122 & A & 96 & & 0.126 \\
\hline
\end{tabular}

*Data of allelic frequencies for the following populations: TSI, Tuscans in Italy; AWS, Americans of African Ancestry; YRI Yorubi in Nigeria.

C. S. Corrent series

\section{HDs vs. BCP within each population}

A1012G polymorphism was equally distributed in HDs and BCPs in both populations. African cases had a higher frequency of AA and TT homozygosity for $\mathrm{Cdx} 2$ and Fok1 SNPs, respectively ( $P=0.02$ in both cases). No significant difference was observed in the Caucasian population (Table 5). We observed no different distribution of haplotype $\mathrm{A} 1012 \mathrm{G} / \mathrm{Cdx} 2$ in either population (Table 3 ).

\section{DBP SNPs}

\section{Africans vs Caucasians}

The rs4588 polymorphism was similarly distributed among African and Caucasian HDs and BCPs. C allele was the most frequent among all subgroups (Table 5). TT homozygotes for rs 7041 were significantly more frequent in Africans HDs $(84 \%$ vs. $18 \%, P<0.0001)$. No African 
Table 5: Distribution of polymorphisms in both populations

\begin{tabular}{|c|c|c|c|c|c|c|}
\hline & \multicolumn{3}{|c|}{ Healthy Donors } & \multicolumn{3}{|c|}{ Patients } \\
\hline & $\begin{array}{l}\text { Africans } \\
\text { No. }(\%)\end{array}$ & $\begin{array}{c}\text { Caucasians } \\
\text { No. }(\%)\end{array}$ & $P$ & $\begin{array}{l}\text { Africans } \\
\text { No. }(\%)\end{array}$ & $\begin{array}{c}\text { Caucasians } \\
\text { No. }(\%)\end{array}$ & $\boldsymbol{P}$ \\
\hline \multicolumn{7}{|l|}{ A1012G } \\
\hline $\mathrm{TT}+\mathrm{CT}$ & $49(98)$ & $45(90)$ & & $18(100)$ & $31(88.6)$ & \\
\hline $\mathrm{CC}$ & $1(2)$ & $5(10)$ & ns & 0 & $4(11.4)$ & ns \\
\hline TT & $45(90)$ & $20(40)$ & & $18(100)$ & $12(34.3)$ & \\
\hline $\mathrm{CT}+\mathrm{CC}$ & $5(10)$ & $30(60)$ & $<0.0001$ & 0 & $23(65.7)$ & $<0.0001$ \\
\hline \multicolumn{7}{|l|}{ Cdx2 } \\
\hline $\mathrm{AA}+\mathrm{GA}$ & $48(96)$ & $24(48)$ & & $18(100)$ & $15(42.9)$ & \\
\hline GG & $2(4)$ & $26(52)$ & $<0.0001$ & 0 & $20(57.1)$ & $<0.0001$ \\
\hline $\mathrm{AA}$ & $39(78)$ & $4(8)$ & & $18(100)$ & $4(11.4)$ & \\
\hline $\mathrm{GA}+\mathrm{GG}$ & $11(22)$ & $46(92)$ & $<0.0001$ & 0 & 31 (88.6) & $<0.0001$ \\
\hline \multicolumn{7}{|l|}{ Fok1 } \\
\hline $\mathrm{TT}+\mathrm{CT}$ & $15(30)$ & $27(54)$ & & $11(61.1)$ & $21(60)$ & \\
\hline $\mathrm{CC}$ & $35(70)$ & $23(46)$ & 0.00253 & 7 (38.9) & $14(40)$ & ns \\
\hline $\mathrm{TT}$ & $1(2)$ & $8(16)$ & & $3(16.7)$ & $5(14.3)$ & \\
\hline $\mathrm{CC}+\mathrm{CT}$ & $49(98)$ & $42(84)$ & 0.03 & $15(83.3)$ & $30(85.7)$ & ns \\
\hline \multicolumn{7}{|l|}{ rs4588 } \\
\hline $\mathrm{AA}+\mathrm{CA}$ & $19(38)$ & $20(40)$ & & $7(38.6)$ & $17(48.6)$ & \\
\hline $\mathrm{CC}$ & $31(62)$ & $30(60)$ & ns & $11(61.1)$ & $18(51.4)$ & ns \\
\hline AA & $0(0)$ & $1(2)$ & & 0 & $2(5.7)$ & \\
\hline $\mathrm{CA}+\mathrm{CC}$ & $50(100)$ & 49 (98) & ns & $18(100)$ & $33(94.3)$ & ns \\
\hline \multicolumn{7}{|l|}{ rs7041 } \\
\hline $\mathrm{GG}+\mathrm{TG}$ & $8(16)$ & $41(82)$ & & $3(16.7)$ & $28(80)$ & \\
\hline TT & $42(84)$ & $9(18)$ & $<0.0001$ & $15(83.3)$ & $7(20)$ & $<0.0001$ \\
\hline GG & $0(0)$ & $16(32)$ & & $0(0)$ & $13(37.1)$ & \\
\hline $\mathrm{TG}+\mathrm{TT}$ & $50(100)$ & $34(68)$ & 0.002 & $18(100)$ & $22(66.0)$ & 0.02 \\
\hline
\end{tabular}

ns $=$ not significant.

Comparison between cases and controls within each population.

The statistically significant comparisons in the African population were: $\mathrm{Cdx} 2 \mathrm{AA}$ vs. $\mathrm{GA}+\mathrm{GG}(P=0.02)$; Fok1: TT $+\mathrm{CTA}$ vs. CC $(P=0.02)$.

No statistically significant comparisons appeared within the Caucasian population.

HD showed GG homozygosity. A similar distribution was observed in BCPs (Table 5).

\section{HDs vs. BCP within each population}

No difference was observed between cases and controls in either African or Caucasian population for rs4588 and rs7041 polymorphisms (Table 5). Haplotypes of rs7041/rs4588 polymorphisms were analyzed only in the Caucasian case series, since the two SNPs reported no Linkage disequilibrium in the African population. The distribution of this haplotype was not significantly different in either Caucasian BCPs or HDs (Table 3).

\section{5(OH)D and DBP levels with respect to SNPs}

\section{VDR SNPs}

We observed no difference in $25(\mathrm{OH}) \mathrm{D}$ and $\mathrm{DBP}$ levels with respect to the allelic variant of the $V D R$ 
Table 6: 25(OH)D and DBP levels according to polymorphism distribution in the African population

\begin{tabular}{|c|c|c|c|c|c|c|c|c|c|c|}
\hline & \multirow{3}{*}{ No. } & \multicolumn{4}{|c|}{ Healthy Donors } & \multicolumn{5}{|c|}{ Patients } \\
\hline & & \multicolumn{2}{|c|}{ 25(OH)D (ng/ml) } & \multicolumn{2}{|l|}{ DBP $(\mu \mathrm{g} / \mathrm{ml})$} & \multicolumn{3}{|c|}{ 25(OH)D (ng/ml) } & \multicolumn{2}{|l|}{$\mathrm{DBP}(\mu \mathrm{g} / \mathrm{ml})$} \\
\hline & & $\begin{array}{l}\text { Median value } \\
\text { (range) }\end{array}$ & $P^{1}$ & $\begin{array}{l}\text { Median value } \\
\text { (range) }\end{array}$ & $\boldsymbol{P}^{1}$ & No. & $\begin{array}{l}\text { Median value } \\
\text { (range) }\end{array}$ & $P^{1}$ & $\begin{array}{l}\text { Median value } \\
\text { (range) }\end{array}$ & $P^{1}$ \\
\hline \multicolumn{11}{|l|}{ A1012G } \\
\hline $\mathrm{TT}+\mathrm{CT}$ & 49 & $23(5-94)$ & & $76.16(31.53-194.75)$ & & 18 & $33.5(14-65)$ & & $87.89(13.69-197.44)$ & \\
\hline $\mathrm{CC}$ & 1 & 17 & 0.252 & 23.34 & 0.096 & 0 & - & - & - & - \\
\hline $\mathrm{TT}$ & 45 & $23(5-94)$ & & $74.11(31.53-194.75)$ & & 18 & $33.5(14-65)$ & & $87.89(13.69-197.44)$ & \\
\hline $\mathrm{CT}+\mathrm{CC}$ & 5 & $18(17-66)$ & 0.935 & $103.67(23.34-119.45)$ & 0.674 & 0 & - & - & - & - \\
\hline \multicolumn{11}{|l|}{$\operatorname{Cdx} 2$} \\
\hline $\mathrm{AA}+\mathrm{GA}$ & 48 & $23(5-94)$ & & $75.13(31.53-194.75)$ & & 18 & $33.5(14-65)$ & & 87.89 (13.69-197.44) & \\
\hline GG & 2 & $17.5(17-18)$ & 0.150 & $63.50(23.34-103.67)$ & 0.569 & 0 & - & - & - & - \\
\hline AA & 39 & $23(9-53)$ & & $73.10(31.53-194.75)$ & & 18 & $33.5(14-65)$ & & 87.89 (13.69-197.44) & \\
\hline $\mathrm{GA}+\mathrm{GG}$ & 11 & $18(5-94)$ & 0.742 & $103.67(23.34-124.76)$ & 0.454 & 0 & - & - & - & - \\
\hline \multicolumn{11}{|l|}{ Fok1 } \\
\hline $\mathrm{TT}+\mathrm{CT}$ & 15 & $23(11-45)$ & & $73.10(23.34-183.08)$ & & 11 & $30(19-46)$ & & $85.29(13.69-197.44)$ & \\
\hline $\mathrm{CC}$ & 35 & $22(5-94)$ & 0.832 & $76.16(31.53-194.75)$ & 0.626 & 7 & $45(14-65)$ & 0.333 & $99.66(33.97-161.05)$ & 0.632 \\
\hline TT & 1 & 18 & & 69.50 & & 3 & $30(28-41)$ & & $84.38(55.11-197.44)$ & \\
\hline $\mathrm{CC}+\mathrm{CT}$ & 49 & $23(5-94)$ & 0.405 & $76.16(23.34-194.75)$ & 0.782 & 15 & $36(14-65)$ & 0.815 & $89.58(13.69-161.05)$ & 0.791 \\
\hline \multicolumn{11}{|l|}{ rs4588 } \\
\hline $\mathrm{AA}+\mathrm{CA}$ & 19 & $23(13-94)$ & & $80.59(36.62-183.08)$ & & 7 & $30(14-46)$ & & 84.38 (48.14-197.44) & \\
\hline $\mathrm{CC}$ & 31 & $21(5-66)$ & 0.477 & $74.11(23.34-194.75)$ & 0.412 & 11 & $36(15-65)$ & 0.594 & $95.56(13.69-161.05)$ & 0.755 \\
\hline AA & 0 & - & & - & & 0 & - & & - & \\
\hline $\mathrm{CA}+\mathrm{CC}$ & 50 & $22.5(5-94)$ & - & $75.13(23.34-194.75)$ & - & 18 & $33.5(14-65)$ & - & $87.89(13.69-194.44)$ & - \\
\hline \multicolumn{11}{|l|}{ rs7041 } \\
\hline $\mathrm{GG}+\mathrm{TG}$ & 8 & $21(9-44)$ & & $100.64(45.01-194.75)$ & & 3 & $26(23-41)$ & & $\begin{array}{c}126.94(117.75- \\
197.44)\end{array}$ & \\
\hline TT & 42 & $23.5(5-94)$ & 0.442 & $72.97(23.34-161.77)$ & 0.078 & 15 & $36(14-65)$ & 0.487 & $84.38(13.69-161.05)$ & 0.048 \\
\hline GG & 0 & - & & - & & 0 & - & & - & \\
\hline $\mathrm{TG}+\mathrm{TT}$ & 50 & $22.5(5-94)$ & - & $75.13(23.34-194.75)$ & - & 18 & $33.5(14-65)$ & - & $87.89(13.69-197.44)$ & - \\
\hline
\end{tabular}

${ }^{1}$ median test.

analyzed polymorphisms in both African HDs and BCPs (Table 6). Caucasian HDs showed no relation between $V D R$ SNPs and the analyzed circulating markers. Patients with CT+ CC alleles for A1012G and Cdx2 polymorphism with GA+GG vs. AA had significantly higher $25(\mathrm{OH}) \mathrm{D}$ levels $(P=0.003$ and $P=0.015$, respectively) (Table 7 ).

\section{DBP SNPs}

African HDs with a G allele in the SNP rs7041 of DBP showed higher levels of DBP - not of 25(OH)D than those with TT homozygosity. Such difference follows the same trend, reaching statistical significance in BCPs $(P=0.048)$ (Table 6). Both Caucasian HDs and BCPs showed about twofold higher levels of DBP with a GG+
TC allele in rs7041. All the comparisons reached statistical significance (Table 7). The presence of CC in rs4588 in HDs and BCPs was related to significantly higher serum levels of DBP $(P=0.010)$ in the Caucasian population (Table 7).

\section{Association of vitamin D and DBP levels with osteoporosis risk factors}

We analyzed serum protein level changes according to osteoporosis risk factors, such as menopausal status, smoking, previous fracture, familiarity, cortisone administration and BMI (Table 8 and 9). Vitamin D levels decreased in Caucasian BC patients with high BMI $(P=$ 0.035). Caucasians HD with previous fractures showed lower DBP levels $(P=0.048)$. 
Table 7: 25(OH)D and DBP levels according to polymorphism distribution in the Caucasian population

\begin{tabular}{|c|c|c|c|c|c|c|c|c|c|c|}
\hline & \multicolumn{5}{|c|}{ Healthy Donors } & \multicolumn{5}{|c|}{ Patients } \\
\hline & & \multicolumn{2}{|c|}{$25(\mathrm{OH}) \mathrm{D}(\mathrm{ng} / \mathrm{ml})$} & \multicolumn{2}{|c|}{$\mathrm{DBP}(\mu \mathrm{g} / \mathrm{ml})$} & \multicolumn{3}{|c|}{$25(\mathrm{OH}) \mathrm{D}(\mathrm{ng} / \mathrm{ml})$} & \multicolumn{2}{|c|}{$\mathrm{DBP}(\mu \mathrm{g} / \mathrm{ml})$} \\
\hline & No. & $\begin{array}{l}\text { Median value } \\
\text { (range) }\end{array}$ & $P^{1}$ & $\begin{array}{l}\text { Median value } \\
\text { (range) }\end{array}$ & $P^{1}$ & No. & $\begin{array}{l}\text { Median value } \\
\text { (range) }\end{array}$ & $\boldsymbol{P}^{1}$ & $\begin{array}{l}\text { Median value } \\
\text { (range) }\end{array}$ & $\boldsymbol{P}^{1}$ \\
\hline \multicolumn{11}{|l|}{ A1012G } \\
\hline $\mathrm{TT}+\mathrm{CT}$ & 45 & $\begin{array}{c}28.15(11.26- \\
52.92)\end{array}$ & & $\begin{array}{c}219.62(101.16- \\
738.06)\end{array}$ & & 31 & $\begin{array}{c}25.94(11.00- \\
53.22)\end{array}$ & & $\begin{array}{c}227.98(79.41- \\
395.70)\end{array}$ & \\
\hline $\mathrm{CC}$ & 5 & $\begin{array}{c}29.76(18.29- \\
60.70)\end{array}$ & 0.698 & $\begin{array}{c}203.41(96.32- \\
304.20)\end{array}$ & 0.654 & 4 & $\begin{array}{c}34.93(32.25- \\
42.70)\end{array}$ & 0.067 & $\begin{array}{c}247.51(154.17- \\
295.76)\end{array}$ & 0.894 \\
\hline TT & 20 & $30.11(11.26-50.63)$ & & $\begin{array}{c}201.02(101.16- \\
738.06)\end{array}$ & & 12 & $\begin{array}{l}20.72(11.00- \\
38.22)\end{array}$ & & $\begin{array}{c}227.98(97.87- \\
385.45)\end{array}$ & \\
\hline $\mathrm{CT}+\mathrm{CC}$ & 30 & $\begin{array}{c}28.11(12.36- \\
60.70)\end{array}$ & 0.586 & $\begin{array}{c}226.04(96.32- \\
485.15)\end{array}$ & 0.806 & 23 & $\begin{array}{c}31.73(19.36- \\
53.22)\end{array}$ & 0.003 & $\begin{array}{c}229.13(79.41- \\
395.70)\end{array}$ & 0.957 \\
\hline \multicolumn{11}{|l|}{ Cdx2 } \\
\hline $\mathrm{AA}+\mathrm{GA}$ & 24 & $\begin{array}{c}28.99(12.36- \\
50.63)\end{array}$ & & $\begin{array}{c}229.50(101.16- \\
738.06)\end{array}$ & & 15 & $\begin{array}{c}25.28(11.00- \\
47.76)\end{array}$ & & $\begin{array}{c}232.20(92.33- \\
385.45)\end{array}$ & \\
\hline GG & 26 & $\begin{array}{c}27.80(11.26- \\
60.70)\end{array}$ & 0.900 & $\begin{array}{c}213.20(96.32- \\
485.15)\end{array}$ & 0.825 & 20 & $\begin{array}{c}30.69(17.66- \\
53.22)\end{array}$ & 0.130 & $\begin{array}{c}221.09(79.41- \\
395.70)\end{array}$ & 0.705 \\
\hline AA & 4 & $\begin{array}{c}34.06(21.86- \\
50.63)\end{array}$ & & $\begin{array}{c}259.66(130.99- \\
369.87)\end{array}$ & & 4 & $\begin{array}{c}13.37(11.00- \\
25.28)\end{array}$ & & $\begin{array}{c}210.09(97.87- \\
326.03)\end{array}$ & \\
\hline $\mathrm{GA}+\mathrm{GG}$ & 46 & $\begin{array}{c}28.32(11.26- \\
60.70)\end{array}$ & 0.734 & $\begin{array}{c}219.62(96.32- \\
738.06)\end{array}$ & 0.682 & 31 & $\begin{array}{c}29.66(14.25- \\
53.22)\end{array}$ & 0.015 & $\begin{array}{c}227.98(79.41- \\
395.70)\end{array}$ & 0.653 \\
\hline \multicolumn{11}{|l|}{ Fok1 } \\
\hline $\mathrm{TT}+\mathrm{CT}$ & 27 & $\begin{array}{c}28.49(11.26- \\
43.24)\end{array}$ & & $\begin{array}{c}201.55(120.85- \\
404.69)\end{array}$ & & 21 & $\begin{array}{l}29.66(14.25- \\
53.22)\end{array}$ & & $\begin{array}{c}216.24(79.41- \\
395.70)\end{array}$ & \\
\hline $\mathrm{CC}$ & 23 & $\begin{array}{c}28.15(12.36- \\
60.70)\end{array}$ & 0.984 & $\begin{array}{c}230.28(96.32- \\
738.06)\end{array}$ & 0.335 & 14 & $\begin{array}{c}23.54(11.00- \\
52.15)\end{array}$ & 0.112 & $\begin{array}{c}233.89(97.87- \\
338.29)\end{array}$ & 0.768 \\
\hline $\mathrm{TT}$ & 8 & $\begin{array}{c}28.49(26.76- \\
37.22)\end{array}$ & & $\begin{array}{c}207.33(133.62- \\
256.55)\end{array}$ & & 5 & $\begin{array}{c}38.22(14.25- \\
47.76)\end{array}$ & & $\begin{array}{c}223.76(166.68- \\
266.59)\end{array}$ & \\
\hline $\mathrm{CC}+\mathrm{CT}$ & 42 & $\begin{array}{c}28.32(11.26- \\
60.70)\end{array}$ & 0.761 & $\begin{array}{c}226.04(96.32- \\
738.06)\end{array}$ & 0.603 & 30 & $\begin{array}{c}26.87(11.00- \\
53.22)\end{array}$ & 0.442 & $\begin{array}{c}232.20(79.41- \\
395.70)\end{array}$ & 0.736 \\
\hline \multicolumn{11}{|l|}{ rs4588 } \\
\hline $\mathrm{AA}+\mathrm{CA}$ & 20 & $\begin{array}{c}27.84(11.26- \\
60.70)\end{array}$ & & $\begin{array}{c}193.47(120.85- \\
267.43)\end{array}$ & & 17 & $\begin{array}{c}25.05(11.00- \\
38.77)\end{array}$ & & $\begin{array}{c}214.90(92.33- \\
326.03)\end{array}$ & \\
\hline $\mathrm{CC}$ & 30 & $\begin{array}{c}28.78(12.36- \\
52.92)\end{array}$ & 0.929 & $\begin{array}{c}251.82(96.32- \\
738.06)\end{array}$ & 0.010 & 18 & $\begin{array}{l}32.31(12.23- \\
53.22)\end{array}$ & 0.022 & $\begin{array}{c}270.88(79.41- \\
395.70)\end{array}$ & 0.134 \\
\hline AA & 1 & 11.26 & & 142.45 & & 2 & $\begin{array}{c}16.94(14.52- \\
19.36)\end{array}$ & & $\begin{array}{c}226.51(127.00- \\
326.03)\end{array}$ & \\
\hline $\mathrm{CA}+\mathrm{CC}$ & 49 & $\begin{array}{c}28.49(12.36- \\
60.70)\end{array}$ & 0.096 & $\begin{array}{c}226.04(96.32- \\
738.06)\end{array}$ & 0.366 & 33 & $\begin{array}{c}28.96(11.00- \\
53.22)\end{array}$ & 0.079 & $\begin{array}{c}227.98(79.41- \\
395.70)\end{array}$ & 1.000 \\
\hline \multicolumn{11}{|l|}{ rs7041 } \\
\hline $\mathrm{GG}+\mathrm{TG}$ & 41 & $\begin{array}{c}28.15(12.36- \\
60.70)\end{array}$ & & $\begin{array}{c}236.46(133.62- \\
738.06)\end{array}$ & & 28 & $\begin{array}{l}29.31(12.23- \\
53.22)\end{array}$ & & $\begin{array}{c}257.40(154.17- \\
395.70)\end{array}$ & \\
\hline TT & 9 & $\begin{array}{c}28.91(11.26- \\
38.33)\end{array}$ & 0.631 & $\begin{array}{c}127.85(96.32- \\
149.02)\end{array}$ & $<0.0001$ & 7 & $\begin{array}{c}24.58(11.00- \\
38.77)\end{array}$ & 0.339 & $\begin{array}{c}100.79(79.41- \\
198.41)\end{array}$ & 0.001 \\
\hline GG & 16 & $\begin{array}{c}29.13(20.69- \\
52.92)\end{array}$ & & $\begin{array}{c}299.34(195.77- \\
738.06)\end{array}$ & & 13 & $\begin{array}{c}32.44(14.52- \\
53.22)\end{array}$ & & $\begin{array}{c}295.76(206.71- \\
395.70)\end{array}$ & \\
\hline $\mathrm{TG}+\mathrm{TT}$ & 34 & $\begin{array}{c}27.49(11.26- \\
60.70)\end{array}$ & 0.499 & $\begin{array}{c}182.30(96.32- \\
278.43)\end{array}$ & $<0.0001$ & 22 & $\begin{array}{c}26.01(11.00- \\
47.76)\end{array}$ & 0.186 & $\begin{array}{c}198.41(79.41- \\
294.16)\end{array}$ & 0.0003 \\
\hline
\end{tabular}

${ }^{1}$ median test. 
Table 8: Median values of 25(OH)D levels according to osteoporosis risk factors

\begin{tabular}{|c|c|c|c|c|}
\hline & \multicolumn{2}{|c|}{ Africans } & \multicolumn{2}{|c|}{ Caucasians } \\
\hline & Healthy & Patients & Healthy & Patients \\
\hline & \multicolumn{4}{|c|}{$\begin{array}{c}25(\mathrm{OH}) \mathrm{D}(\mathrm{ng} / \mathrm{ml}) \\
\text { Median value (range) }\end{array}$} \\
\hline \multicolumn{5}{|l|}{ Menopausal status } \\
\hline Premenopausal & $22(5-94)$ & $31(14-65)$ & $28.32(11.26-60.70)$ & $31.49(25.94-40.48)$ \\
\hline Postmenopausal & $27(21-66)$ & $36(15-48)$ & $27.39(12.36-34.79)$ & $24.81(11.00-52.15)$ \\
\hline \multicolumn{5}{|l|}{ Smoking habits } \\
\hline No & $22.5(5-94)$ & $33.5(14-65)$ & $28.70(14.34-52.92)$ & $28.96(12.23-53.22)$ \\
\hline Yes & - & - & $24.98(11.26-60.70)$ & $25.66(11.00-38.22)$ \\
\hline \multicolumn{5}{|l|}{ Fractures } \\
\hline No & $22.5(5-94)$ & $33.5(14-65)$ & $28.07(12.36-60.70)$ & $26.74(12.23-40.48)$ \\
\hline Yes & - & - & $28.97(11.26-43.24)$ & $23.91(11.00-52.15)$ \\
\hline \multicolumn{5}{|l|}{$\begin{array}{l}\text { Familiar } \\
\text { predisposition }\end{array}$} \\
\hline No & $22.5(5-94)$ & $33.5(14-65)$ & $28.91(11.26-60.70)$ & $26.34(11.00-52.15)$ \\
\hline Yes & - & - & $26.76(12.36-41.47)$ & $25.05(-)$ \\
\hline \multicolumn{5}{|l|}{ Corticosteroid use } \\
\hline No & $22.5(5-94)$ & $33.5(14-65)$ & $28.49(11.26-60.70)$ & $25.61(11.00-52.15)$ \\
\hline Yes & - & - & - & $29.93(22.78-37.42)$ \\
\hline \multicolumn{5}{|l|}{ BMI } \\
\hline$<20$ & - & - & $29.18(12.36-50.63)$ & $28.96(14.25-47.76)$ \\
\hline $20-25$ & $23(5-94)$ & $38.5(14-65)$ & $28.32(11.26-60.70)$ & $29.93(21.39-52.15)$ \\
\hline$>25$ & $11(-)$ & $21(19-23)$ & $27.13(18.29-41.47)$ & $20.06(11.00-42.70)$ \\
\hline
\end{tabular}

All $\mathrm{p}$ were $>0.05$ except for the comparison of $25(\mathrm{OH}) \mathrm{D}$ differences according to BMI in Caucasian patients.

\section{DISCUSSION}

We performed a study comparing the vitamin D pathway status in a native black Tanzanian and a Caucasian Italian population in an $\mathrm{HD}$ and $\mathrm{BCP}$ series. We studied 25(OH)D and DBP levels, together with a number of polymorphisms of $V D R$ and $D B P$ for better understanding the degree of activation of the vitamin $\mathrm{D}$ pathway in both populations.

Powe published data from a large case series regarding black and white Americans, reporting DBP levels twofold lower in Blacks than in Whites; our data showed a threefold change [29]. This discrepancy could be due to the differences between African Americans and our Tanzanian population in the case series size, median age (as individuals in Powe's case series were older), gender (as not all individuals were women), provenance and daily habits, such as diet and smoking.
Powe reported that lower DBP levels could explain the coexistence in the black population of low levels of vitamin D and the absence of any skeletal problems. This theory is in line with the previously reported hypothesized role of DBP as regulator of vitamin D [18, 19], by which low levels of DBP can counterbalance low levels of vitamin D. Some authors recently reported that besides entering the cells as an unbound hormone, vitamin D can enter cells bound to DBP through an endocytosis uptake mechanism [36-37]. Further studies are needed to understand whether this new discovery impacts the role of DBP in vitamin $D$ regulation.

The DBP polymorphisms rs7041 and rs4588 analyzed by Powe and in the present study were related to racial differences in circulating DBP levels [29]. In particular, $\mathrm{T}$ allele was more frequent and $\mathrm{A}$ allele was less frequent in Blacks at rs7041 and rs4588, respectively. These variants were related to lower and higher DBP 
Table 9: Median values DBP levels according to osteoporosis risk factors

\begin{tabular}{|c|c|c|c|c|}
\hline & \multicolumn{2}{|c|}{ Africans } & \multicolumn{2}{|c|}{ Caucasians } \\
\hline & Healthy & Patients & Healthy & Patients \\
\hline & \multicolumn{4}{|c|}{$\begin{array}{c}\text { DBP_( } \mu \mathrm{g} / \mathrm{ml}) \\
\text { Median value (range) }\end{array}$} \\
\hline \multicolumn{5}{|l|}{ Menopausal status } \\
\hline Premenopausal & $75.13(23.34-194.75)$ & $84.38(13.69-197.44)$ & $219.62(96.32-738.06)$ & $265.25(103.72-395.70)$ \\
\hline Postmenopausal & $78.59(53.58-119.45)$ & $117.75(33.97-161.05)$ & $211.48(127.85-299.34)$ & $208.05(79.41-385.45)$ \\
\hline \multicolumn{5}{|l|}{ Smoking habits } \\
\hline No & $75.13(23.34-194.75)$ & 87.89 (13.69-197.44) & $226.04(96.32-738.06)$ & $226.64(92.33-395.70)$ \\
\hline Yes & - & - & $193.47(122.53-404.69)$ & 236.65 (79.41-295.76) \\
\hline \multicolumn{5}{|l|}{ Fractures } \\
\hline No & $75.13(23.34-194.75)$ & $87.89(13.69-197.44)$ & $236.46(96.32-738.06)$ & 242.57 (79.41-395.70) \\
\hline Yes & - & - & $179.03(127.85-231.07)$ & $202.56(92.33-385.45)$ \\
\hline \multicolumn{5}{|l|}{ Familiarity } \\
\hline No & $75.13(23.34-194.75)$ & $87.89(13.69-197.44)$ & $213.20(96.32-738.06)$ & $223.76(79.41-395.70)$ \\
\hline Yes & - & - & $232.98(101.16-293.92)$ & $208.72(-)$ \\
\hline \multicolumn{5}{|l|}{ Cortisone } \\
\hline No & $75.13(23.34-194.75)$ & 87.89 (13.69-197.44) & $226.04(96.32-738.06)$ & $208.72(79.41-395.70)$ \\
\hline Yes & - & - & - & $290.52(92.33-295.76)$ \\
\hline \multicolumn{5}{|l|}{ BMI } \\
\hline$<20$ & - & - & $186.26(122.53-337.03)$ & $223.98(79.41-395.70)$ \\
\hline $20-25$ & $74.11(23.34-194.75)$ & $85.29(13.69-197.44)$ & $230.30(96.32-738.06)$ & $235.59(154.17-338.29)$ \\
\hline$>25$ & $76.81(-)$ & $111.25(95.56-126.94)$ & $236.46(175.76-299.34)$ & $186.08(97.87-385.45)$ \\
\hline
\end{tabular}

All $\mathrm{p}$ were $>0.05$ except for the comparison of DBP differences according to the presence of fractures in Caucasian HDs.

levels [29], respectively. Our data showed a higher prevalence among Blacks of rs 7041 polymorphism in the $D B P$ gene associated with low levels of DBP, potentially resulting in bioavailable $25(\mathrm{OH}) \mathrm{D}$ levels similar to those in Whites, despite their lower levels of total 25(OH)D.

Unlike Powe et al., we analyzed a number of SNPs related to $V D R$, such as $\mathrm{A} 1012 \mathrm{G}, \mathrm{Cdx} 2$, and Fok1. The first and the second are located in the promoter regions of the $V D R$, which could influence $V D R$ expression [38-39].

$\mathrm{G}$ allele of $\mathrm{Cdx} 2$ decreases $V D R$ gene transcription. Fok1 polymorphism, instead, produces two alleles distinguished by the presence or absence of the Fok1 restriction site. TT allele encodes full-length VDR protein, while CC allele encodes a shorter protein. Our data showed that the Tanzanian population was more likely to have Fok1 CC homozygosity, which resulted in a VDR protein with higher transcriptional activity, and $\mathrm{Cdx} 2 \mathrm{AA}$ homozygosity, related to an increase in the transcription of VDR gene. Considering the differences in allelic variant distribution between cases and controls within the African population, we interestingly observed that BCPs and HDs have $38 \%$ and $70 \%$ of CC for Fok 1 SNP, respectively. Given that vitamin D could act as a shield against BC development [10-15] since $\mathrm{CC}$ is related to a higher activity of VDR, this datum suggests that the vitamin D pathway status can play a protective role against BC development in Africans.

A number of papers, including several meta analyses reporting data on thousands of individuals, evaluated the role of Fok1 and Cdx2 and other VDR polymorphisms in $\mathrm{BC}$ risk increase with mixed results [40-50]. Our series is still overly limited to allow for a $\mathrm{BC}$ risk analysis.

Our paper has few limitations: first, the small case series size impeded any statistical adjustment according to population characteristics and time of the year of sample collection. In fact, Caucasians were all enrolled in the same period, whereas Tanzania's lower latitude and consistent sunlight exposure made the time of collection 
Table 10: Characteristics of BCPs and HDs in the African and Caucasian populations

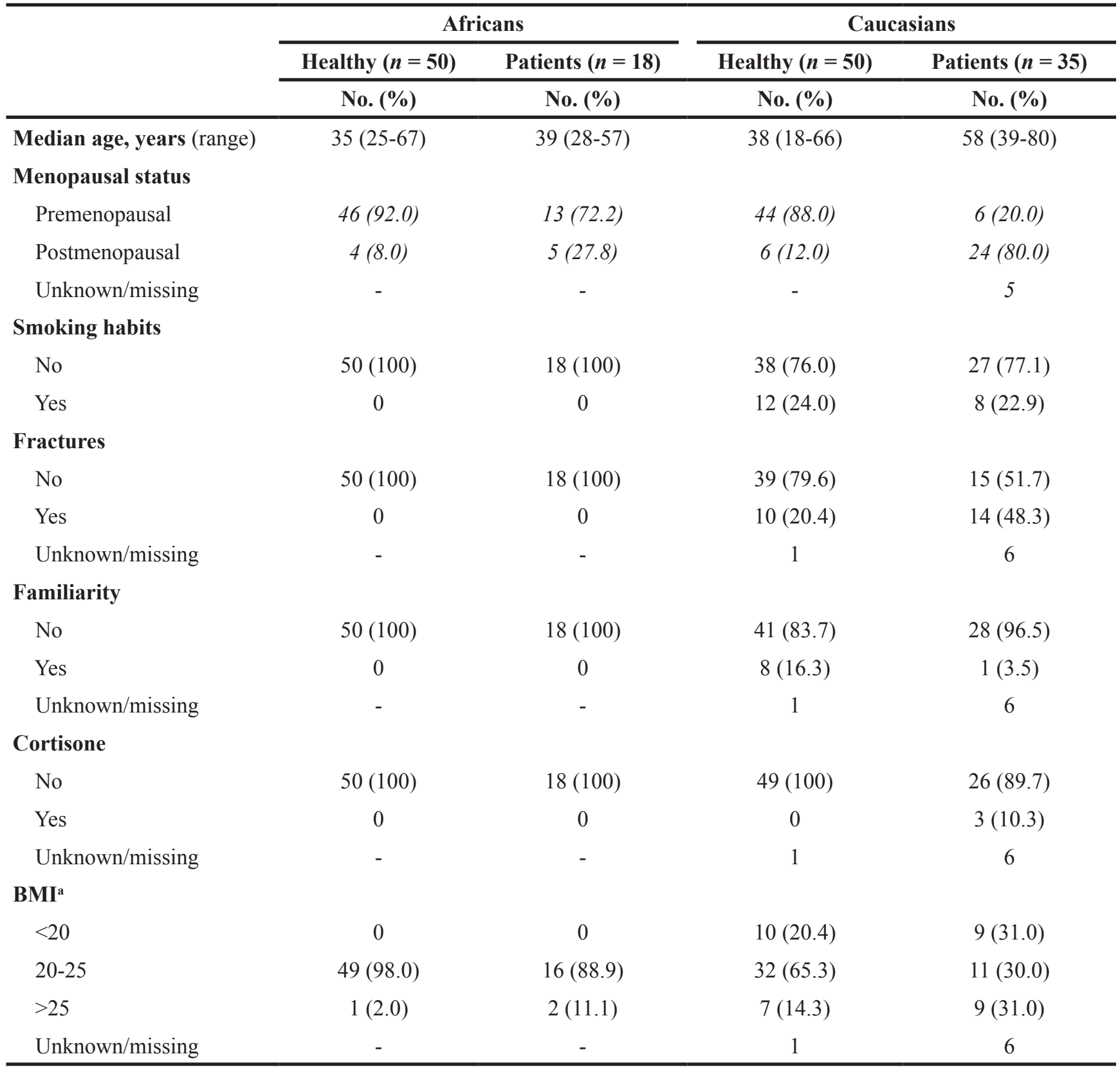

aBMI, body mass index.

less relevant. Secondly, HDs and $\mathrm{BCPs}$ of the two populations were not matched by age: African BCPs were younger than Caucasians BCPs, as Africans developed BC earlier than Caucasians [27, 51].

Our results demonstrated that the two analyzed populations are different not only with respect to DBP levels, but also to the allelic variant distribution of $V D R$ and $D B P$ SNPs. According to our results, Africans have innate higher levels and stronger activity of VDR, with a consequent higher degree of activation of the vitamin D pathway. These alterations, alongside those in DBP levels previously reported in literature, may be responsible for the observed racial differences in the total vitamin
D levels in absence of symptomatic manifestations of vitamin D deficiency in Blacks [29]. So far a lower BC incidence has been observed in Africans and African Americans than in white Americans, even if $\mathrm{BC}$ incidence in African Americans is currently rising [27, 52]. Although these differences also stem from several genetic and environmental factors, we hypothesize that one of the mechanisms involved is that Africans, despite vitamin D deficiency, can be protected from BC thanks to a higher activity of the vitamin $\mathrm{D}$ pathway.

Although the hypothesized mechanism awaits to be confirmed, it nevertheless elicits further investigation on genetic racial differences with respect to cancer. 
Table 11: Characteristics of BCPs in the African and Caucasian populations

\begin{tabular}{|c|c|c|}
\hline & \multicolumn{2}{|c|}{ Patients } \\
\hline & African $(n=18)$ & Caucasian $(n=35)$ \\
\hline & No. $(\%)$ & No. $(\%)$ \\
\hline \multicolumn{3}{|l|}{ Stage } \\
\hline I & 0 & $16(45.7)$ \\
\hline II & 0 & $12(34.3)$ \\
\hline III & $3(16.7)$ & $1(2.9)$ \\
\hline IV & $2(11.1)$ & 0 \\
\hline Unknown/missing & $13(72.2)$ & $6(17.1)$ \\
\hline \multicolumn{3}{|l|}{ PgR } \\
\hline Negative $(<10 \%)$ & - & $10(33.3)$ \\
\hline Positive $(\geq 10 \%)$ & - & $20(66.7)$ \\
\hline Unknown/missing & 18 & 5 \\
\hline \multicolumn{3}{|l|}{ HER2 } \\
\hline Negative & - & $27(90.0)$ \\
\hline Positive & - & $3(10.0)$ \\
\hline Unknown/missing & 18 & 5 \\
\hline \multicolumn{3}{|l|}{ Mib1 } \\
\hline Negative $(\leq 20 \%)$ & - & $20(66.7)$ \\
\hline Positive $(>20 \%)$ & - & $10(33.3)$ \\
\hline Unknown/missing & 18 & 5 \\
\hline \multicolumn{3}{|c|}{ Neoadjuvant chemotherapy } \\
\hline No & - & $29(96.7)$ \\
\hline Yes & - & $1(3.3)$ \\
\hline Unknown/missing & 18 & 5 \\
\hline \multicolumn{3}{|l|}{ Surgery } \\
\hline No & 0 & $1(3.3)$ \\
\hline Yes & $1(5.6)$ & $29(96.7)$ \\
\hline Unknown/missing & 17 & 5 \\
\hline \multicolumn{3}{|l|}{ Hormone therapy } \\
\hline Adjuvant & 0 & $25(83.3)$ \\
\hline Advanced & $12(66.7)$ & $3(10.0)$ \\
\hline Unknown/missing & - & 5 \\
\hline \multicolumn{3}{|l|}{ Chemotherapy } \\
\hline Adjuvant & 0 & $11(36.7)$ \\
\hline Advanced & $17(94.4)$ & $2(6.7)$ \\
\hline Unknown/missing & - & 5 \\
\hline \multicolumn{3}{|l|}{ Radiotherapy } \\
\hline Adjuvant & 0 & $23(76.7)$ \\
\hline Advanced & $2(11.1)$ & 0 \\
\hline Unknown/missing & - & 5 \\
\hline
\end{tabular}




\section{MATERIALS AND METHODS}

\section{Study design}

This prospective study was carried out at the Istituto Scientifico Romagnolo per lo Studio e la Cura dei Tumori (IRST) IRCCS (Meldola, Italy) in collaboration with the Bugando Medical Centre (Mwanza, Tanzania). Our primary objective was to compare $25(\mathrm{OH}) \mathrm{D}$ levels in an African and a Caucasian population of HDs and BCPs. We aimed to observe differences between the populations, and between cases and controls. We investigated also other markers of the vitamin D pathway, such as DBP levels and 5 polymorphisms (SNPs) of $V D R$ (FokI, Cdx2 and $\mathrm{A} 1012 \mathrm{G}$ ) and $D B P$ (rs4588 and rs7041), correlating them with the vitamin $\mathrm{D}$ trend and the $\mathrm{BC}$ biological features of the African and Caucasian BCPs. The protocol was reviewed and approved by the local ethics committee and performed according to the Good Clinical Practice and the Declaration of Helsinki. The patients gave their written informed consent to take part in the study.

\section{Case series}

Peripheral venous blood (PB) samples were obtained from 85 Caucasian (50 HDs and 35 BCPs) and 68 African women (50 HDs and 18 BCPs). HDs and BCPs were enrolled between 2014 and 2015. HDs and BCPs were eligible if not taking supplementation of vitamin D. Case series details are reported in Tables 10 and 11.

\section{Biological samples}

For each HD and BCP 2 tubes of PB were collected: one $5 \mathrm{ml}$ tube without anticoagulant and one $3 \mathrm{ml}$ tube with EDTA. The first tube was left to coagulate for 30 minutes at room temperature and centrifuged at 2,000 $\mathrm{g}$ for 15 minutes, after which serum was stored at $-80^{\circ} \mathrm{C}$ until assays were performed. Blood from the second tube was aliquoted and stored at $-20^{\circ} \mathrm{C}$ until DNA extraction. Each blood and serum sample was also stored in Whatman filter paper (Milan, Italy) for dried blood spot (DBS) and dried serum spot (DSS) sampling. Briefly, $50 \mu \mathrm{l}$ of serum/entire blood were spotted in 5 consecutive filters of a Whatman paper card allowed to dry overnight and stored in a plastic bag with desiccant at $-20^{\circ} \mathrm{C}$ until use.

\section{5(OH)D levels}

Serum 25(OH)D level is currently the best representative form of vitamin $\mathrm{D}$ for measuring vitamin D status. For the Caucasian population levels of $25(\mathrm{OH})$ D were quantified in serum by an IVD commercial immunoenzymatic assay by Biovendor (Hamburg, Germany) following the manufacturer's instructions. For the African population, 25(OH)D levels were quantified by serum spotted in filters by ZRT Laboratory using liquid chromatography/tandem mass spectrometry (LC-MS/MS), which is also used by the CDC Nutrition Laboratory to obtain accurate $25(\mathrm{OH}) \mathrm{D}$ values [53]. Before merging the vitamin D levels obtained with the 2 methods, we tested the reproducibility of the 2 assays analyzing 13 samples with both methods. Results were considered satisfactory as the coefficient variation was $<15 \%$ in all the comparisons. The 25(OH)D levels observed included free and bound vitamin $\mathrm{D}$.

\section{DBP levels}

DBP levels were evaluated by a commercial immunoenzymatic kit (R\&D system) starting from DSS stored as described above. One spot for each sample was allowed to thaw, cut in small pieces and put into a $1.5 \mathrm{ml}$ tube with $400 \mu 1$ of PBS. Samples were shaken overnight at $4^{\circ} \mathrm{C}$ [54]. The protein eluate obtained was utilized as a serum sample for DBP detection according to the manufacturer's instructions, after appropriate dilutions.

\section{Genotyping}

Genomic DNA from the Caucasian population was extracted from PB samples by QIAamp DNA Mini Kit (Qiagen, Hilden, Germany) according to the Blood and Body Fluid Spin protocol. Genomic DNA from the African population was instead extracted by QIAmp DNA micro kit according to the Dried Blood Spot protocol. DNA quantity and quality were assessed by Nanodrop 1000 (Celbio, Milan, Italy). Fifteen ng of DNA were used to detect each SNPs by real-time PCR using TaqMan SNP assays and Taqman genotyping Master mix ThermoFisher Scientific in a total volume of $10 \mu \mathrm{l}$ according to the manufacturer's instructions. PCR reaction consisted in: 10 minutes at $95^{\circ} \mathrm{C}, 15$ seconds at $95^{\circ} \mathrm{C}$ for DNA denaturation, and 1 minute at $60^{\circ} \mathrm{C}$ for annealing and extension for 40 cycles.

The real-time PCR profiles of homo- and heterozygosis of Rs7041 were not clear. For this reason 10 additional samples were analyzed only for Rs 7041 by direct sequencing to confirm real-time PCR (primer sequences: F: TCGAAGAGGCATGTTTCACT; R:GCAGTTGGA GGCAAAGTCTG).

After sequencing and genotyping, the HardyWeinberg equilibrium was determined by the Haploview program (Version 4.2) (Broad Institute of MIT and Harvard University).

\section{Statistical analyses}

Descriptive analyses were presented for demographic and clinical characteristics. Categorical variables were presented as numbers and percentages, and continuous data as median and range. The distribution of serum vitamin $\mathrm{D}$ levels was categorized as deficient $(<20 \mathrm{ng} / \mathrm{mL}$ ), suboptimal (20 to $29 \mathrm{ng} / \mathrm{mL}$ ), optimal 
(30.50 ng/mL), upper-normal and above (> $50 \mathrm{ng} /$ $\mathrm{mL})$. The DBP levels were categorized according to the tertile of distribution. Differences between populations, between vitamin-D-deficient and non-vitamin-Ddeficient women with respect to the demographic and clinical characteristics, and between allelic frequency of SNP in our Caucasian and African case series and in a 100-genome dataset were examined with the Chi-square test. The Spearman's correlation was used to assess the correlations between vitamin D and DBP levels considered as continuous variables. The nonparametric median test was used to estimate the correlation between the continuous variables in the two populations (African or Caucasian) or subject status (HD or BCP). All statistical analyses were conducted with SAS Statistical software, version 9.4 (SAS Institute).

\section{ACKNOWLEDGMENTS}

We would like to thank Veronica Zanoni and Laura Lotito for editorial assistance.

\section{CONFLICTS OF INTEREST}

The authors have no conflicts of interest to declare.

\section{Authors' contributions}

Conception, interpretation of data and writing of the manuscript: Dino Amadori, Patrizia Serra and Laura Mercatali.

Patient enrolment: Nestory Masalu, Aloyce Maria Bugingo, Toni Ibrahim and Alberto Bongiovanni.

HD enrolment: Valentina Turri and Rosanna Tedaldi.

Data acquisition: Giacomo Miserocchi, Chiara Spadazzi, Chiara Liverani and Deogratias Katabalo.

Storage of African biological samples and contribution to experimental results: Akwilina Pangan.

Analysis and interpretation of data: Emanuela Scarpi.

All authors approved the final version of the manuscript.

\section{REFERENCES}

1. Institute of Medicine (US) Committee to Review Dietary Reference Intakes for Vitamin D and Calcium. In: Ross AC, Taylor CL, Yaktine AL, Del Valle HB, editors). Dietary reference intakes for calcium and vitamin D. National Academies Press (US); 2011.

2. Gutiérrez OM, Farwell WR, Kermah D, Taylor EN. Racial differences in the relationship between vitamin $\mathrm{D}$, bone mineral density, and parathyroid hormone in the National
Health and Nutrition Examination Survey. Osteoporos Int. 2011; 22:1745-1753.

3. Ginde AA, Liu MC, Camargo CA Jr. Demographic differences and trends of vitamin D insufficiency in the US population, 1988-2004. Arch Intern Med. 2009; 169:626-632.

4. Hannan MT, Litman HJ, Araujo AB, McLennan CE, McLean RR, McKinlay JB. Serum 25-hydroxyvitamin D and bone mineral density in a racially and ethnically diverse group of men. J Clin Endocrinol Metab. 2008; 93:40-46.

5. Cauley JA, Lui LY, Ensrud KE, Zmuda JM, Stone KL, Hochberg MC. Bone mineral density and the risk of incident nonspinal fractures in black and white women. JAMA. 2005; 293:2102-2108.

6. Feldman D, Krishnan AV, Swami S, Giovannucci E, Feldman BJ. The role of vitamin D in reducing cancer risk and progression. Nat Rev Cancer. 2014; 14:342-357.

7. Dusso AS1, Brown AJ, Slatopolsky E. Vitamin D. Am J Physiol Renal Physiol. 2005 Jul; 2891:F8-28.

8. Bikle DD. Vitamin D metabolism, mechanism of action, and clinical applications. Chem Biol. 2014; 21:319-329.

9. Narvaez CJ, Matthews D, LaPorta E, Simmons KM, Beaudin S, Welsh J. The impact of vitamin D in breast cancer: genomics, pathways, metabolism. Front Physiol. 2014; 5:213.

10. Giovannucci E, Liu Y, Rimm EB, Hollis BW, Fuchs CS, Stampfer MJ. Prospective study of predictors of vitamin D status and cancer incidence and mortality in men. J Natl Cancer Inst. 2006; 98:451-459.

11. Kühn T, Kaaks R, Becker S, Eomois PP, Clavel-Chapelon F, Kvaskoff M. Plasma 25-hydroxyvitamin D and the risk of breast cancer in the European prospective investigation into cancer and nutrition: a nested case-control study. Int J Cancer. 2013; 133:1689-1700.

12. Bauer SR, Hankinson SE, Bertone-Johnson ER, Ding EL. Plasma vitamin D levels, menopause, and risk of breast cancer: dose-response meta-analysis of prospective studies. Medicine. 2013; 92:123-131.

13. Wang D, Vélez de-la-Paz OI, Zhai JX, Liu DW. Serum 25-hydroxyvitamin $\mathrm{D}$ and breast cancer risk: a metaanalysis of prospective studies. Tumour Biol. 2013; 34:3509-3517.

14. Chung M, Lee J, Terasawa T, Lau J, Trikalinos TA. Vitamin $\mathrm{D}$ with or without calcium supplementation for prevention of cancer and fractures: an updated meta-analysis for the U.S. Preventive Services Task Force. Ann Intern Med. 2011; 155:827-838.

15. Manson JE, Mayne ST, Clinton SK. Vitamin D and prevention of cancer - ready for prime time? N Engl J Med. 2011; 364:1385-1388.

16. Jacot W, Firmin N, Roca L, Topart D, Gallet S, Durigova A, Mirr S, Abach L, Pouderoux S, D'Hondt V, Bleuse JP, Lamy PJ, Romieu G. Impact of a tailored oral vitamin D 
supplementation regimen on serum 25-hydroxyvitamin D levels in early breast cancer patients: a randomized phase III study. Ann Oncol. 2016; 27:1235-1241.

17. Bouillon B. The vitamin D binding protein, in Feldman D, Pike JW, Adams J, editors. Vitamin D. London, Elsevier. 2011. pp. 57-72.

18. Safadi FF, Thornton P, Magiera H, Hollis BW, Gentile M, Haddad JG, Liebhaber SA, Cooke NE. Osteopathy and resistance to vitamin $\mathrm{D}$ toxicity in mice null for vitamin $\mathrm{D}$ binding protein. J Clin Invest 1999; 103:239-51. 21.

19. Bikle DD, Gee E. Free, and not total, 1,25-dihydroxyvitamin $\mathrm{D}$ regulates 25- hydroxyvitamin $\mathrm{D}$ metabolism by keratinocytes. Endocrinology 1989;124:649-54. New

20. Feldman D, Krishnan AV, Swami S. In: Marcus R, Feldman D, Dempster D, Luckey M, Cauley J. Osteoporosis. 4th ed. San Diego CA: Elsevier Academic Press. 2013; p. 283-329.

21. Haussler MR, Whitfield GK, Kaneko I, Haussler CA, Hsieh D, Hsieh JC. Molecular mechanisms of vitamin D action. Calcif Tissue Int. 2013; 92:77-98.

22. Krishnan AV, Feldman D. Mechanisms of the anti-cancer and anti-inflammatory actions of vitamin D. Annu Rev Pharmacol Toxicol. 2011; 51:311-336.

23. Arnaud J, Constans J. Affinity differences for vitamin D metabolites associated with the genetic isoforms of the human serum carrier protein (DBP). Hum Genet. 1993; 92:183-188.

24. Braun A, Bichlmaier R, Cleve H. Molecular analysis of the gene for the human vitamin-D-binding protein (groupspecific component): allelic differences of the common genetic GC types. Hum Genet. 1992; 89:401-406.

25. Engelman $\mathrm{CD}$, Fingerlin TE, Langefeld $\mathrm{CD}$, Hicks PJ, Rich SS, Wagenknecht LE. Genetic and environmental determinants of 25-hydroxyvitamin D and 1,25-dihydroxyvitamin D levels in Hispanic and Africans Americans. J Clin Endocrinol Metab. 2008; 93:3381-3388.

26. Constans J, Hazout S, Garruto RM, Gajdusek DC, Spees EK. Population distribution of the human vitamin D binding protein: anthropological considerations. Am J Phys Anthropol. 1985; 68:107-122.

27. Ijaduola TG, Smith EB. Pattern of breast cancer among white-American, African-American, and nonimmigrant west-African women. J Natl Med Assoc. 1998; 90:547-551.

28. Amadori D, Serra P, Bravaccini S, Farolfi A, Puccetti M, Carretta E. Differences in biological features of breast cancer between Caucasian (Italian) and African (Tanzanian) populations. Breast Cancer Res Treat. 2014; 145:177-183.

29. Powe CE, Evans MK, Wenger J, Zonderman AB, Berg AH, Nalls M, Tamez H. Vitamin D-binding protein and vitamin D status of black Americans and white Americans. N Engl J Med. 2013; 369:1991-2000.

30. Grant DJ, Hoyo C, Akushevich L, Iversen ES, Whitaker R, Marks J. Vitamin D receptor (VDR) polymorphisms and risk of ovarian cancer in Caucasian and African American women. Gynecol Oncol 2013; 129:173-178.
31. Mishra DK, Wu Y, Sarkissyan M, Sarkissyan S, Chen Z, Shang X. Vitamin D receptor gene polymorphisms and prognosis of breast cancer among Africans-American and Hispanic women. PLoS One. 2013; 8:e57967.

32. O Neill V, Asani FF, Jeffery TJ, Saccone DS, Bornman L. Vitamin D receptor gene expression and function in a south africans population: ethnicity, vitamin D and FokI. PLoS One. 2013; 8:e67663.

33. Kramer H, Camacho P, Aloia J, Luke A, Bovet P, Rhule JP. Association between 25-hydroxyvitamin D and intact parathyroid hormone levels across latitude among adults with African ancestry. Endocr Pract. 2016; 22:911-919.

34. Amadori D, Serra P, Bucchi L, Altini M, Majinge C, Kahima J. The Mwanza Cancer Project. Lancet Oncol. 2016; 17:146-148.

35. New A global reference for human genetic variation, The 1000 Genomes Project Consortium, Nature 526, 68-74 (01 October 2015) doi:10.1038/nature15393.

36. Rowling MJ, Kemmis CM, Taffany DA, Welsh J. Megalinmediated endocytosis of vitamin D binding protein correlates with 25-hydroxycholecalciferol actions in human mammary cells. J Nutr. 2006; 136:2754-9.

37. Nykjaer A1, Dragun D, Walther D, Vorum H, Jacobsen C, Herz J, Melsen F, Christensen EI, Willnow TE. An endocytic pathway essential for renal uptake and activation of the steroid 25-(OH) vitamin D3. Cell. 1999; 96:507-15.

38. Jehan F, d'Alésio A, Garabédian M. Exons and functional regions of the human vitamin $\mathrm{D}$ receptor gene around and within the main 1a promoter are well conserved among mammals. J Steroid Biochem Mol Biol. 2007; 103:361-367.

39. Halsall A, Osborne JE, Potter L, Pringle JH, Hutchinson PE. A novel polymorphism in the 1A promoter region of the vitamin $\mathrm{D}$ receptor is associated with altered susceptibilty and prognosis in malignant melanoma. Br J Cancer. 2004; 91:765-770.

40. Engel LS, Orlow I, Sima CS, Satagopan J, Mujumdar U, Roy P. Vitamin D receptor gene haplotypes and polymorphisms and risk of breast cancer: a nested casecontrol study. Cancer Epidemiol Biomarkers Prev. 2012; 21:1856-1667.

41. Chen WY, Bertone-Johnson ER, Hunter DJ, Willett WC, Hankinson SE. Associations between polymorphisms in the vitamin D receptor and breast cancer risk. Cancer Epidemiol Biomarkers Prev. 2005; 14:2335-2339.

42. Barroso E, Fernandez LP, Milne RL, Pita G, Sendagorta E, Floristan U. Genetic analysis of the vitamin $\mathrm{D}$ receptor gene in two epithelial cancers: melanoma and breast cancer casecontrol studies. BMC Cancer 2008; 8:385.

43. Gapska P, Scott RJ, Serrano-Fernandez P, Huzarski T, Byrski T, Kladny J. Vitamin D receptor variants and breast cancer risk in the Polish population. Breast Cancer Res Treat. 2009; 115:629-633. 
44. McKay JD, McCullough ML, Ziegler RG, Kraft P, Saltzman BS, Riboli E. Vitamin D receptor polymorphisms and breast cancer risk: results from the National Cancer Institute Breast and Prostate Cancer Cohort Consortium. Cancer Epidemiol Biomarkers Prev. 2009; 18:297-305

45. Anderson LN, Cotterchio M, Cole DE, Knight JA. Vitamin D-related genetic variants, interactions with vitamin D exposure, and breast cancer risk among Caucasian women in Ontario. Cancer Epidemiol Biomarkers Prev. 2011; 20:1708-1717.

46. Huang QQ, Liao YY, Ye XH, Fu JJ, Chen SD. Association between VDR polymorphisms and breast cancer: an updated and comparative meta-analysis of crude and adjusted odd ratios. Asian Pac J Cancer Prev. 2014; 15:847-853.

47. Serrano D, Gnagnarella P, Raimondi S, Gandini S. Metaanalysis on vitamin $\mathrm{D}$ receptor and cancer risk: focus on the role of TaqI, ApaI, and Cdx2 polymorphisms. Eur J Cancer Prev. 2016; 25:85-96.

48. Lurie G, Wilkens LR, Thompson PJ, McDuffie KE, Carney ME, Terada KY. Vitamin D receptor gene polymorphisms and epithelial ovarian cancer risk. Cancer Epidemiol Biomarkers Prev. 2007; 16:2566-2571

49. Huang J, Huang J, Ma Y, Wang H, Yang J, Xiong T. The $\mathrm{Cdx}-2$ polymorphism in the VDR gene is associated with increased risk of cancer: a meta-analysis. Mol Biol Rep. 2013; 40:4219-4225.

50. Yao S, Zirpoli G, Bovbjerg DH, Jandorf L, Hong CC, Zhao H. Variants in the vitamin D pathway, serum levels of vitamin D, and estrogen receptor negative breast cancer among african-american women: a case-control study. Breast Cancer Res. 2012; 14:R58.

51. Harris JR, Lippman ME, Veronesi U, Willett W. Breast cancer. N Engl J Med1992; 327:319-28. 47.

52. Jemal A, Siegel R, Xu J, Ward E. Cancer statistics, 2010. CA Cancer J Clin. 2010; 60:277-300.

53. Newman MS, Brandon TR, Groves MN, Gregory WL, Kapur S, Zava DT. A liquid chromatography/tandem mass spectrometry method for determination of 25-hydroxy vitamin D2 and 25-hydroxy vitamin D3 in dried blood spots: a potential adjunct to diabetes and cardiometabolic risk screening. J Diabetes Sci Technol. 2009; 31:156-162.

54. Colson KE, Potter A, Conde-Glez C, Hernandez B, RíosZertuche D, Zúñiga-Brenes P. Use of a commercial ELISA for the detection of measles-specific immunoglobulin $G$ (IgG) in driedblood spots collected from children living in low-resource settings. J Med Virol. 2015; 87:1491-1499. 